\title{
COMPARISON OF MILLIMETER-WAVE AND X-RAY EMISSION IN SEYFERT GALAXIES
}

\author{
R. R. Monje, A. W. Blain, and T. G. Phillips \\ Division of Physics, Mathematics and Astronomy, California Institute of Technology, Pasadena, CA 91125-4700, USA \\ Received 2010 January 7; accepted 2011 June 20; published 2011 August 4
}

\begin{abstract}
We compare the emission at multiple wavelengths of an extended Seyfert galaxy sample, including both types of Seyfert nuclei. We use the Caltech Submillimeter Observatory to observe the CO $J=2-1$ transition line in a sample of 45 Seyfert galaxies and detect 35 of them. The galaxies are selected by their joint soft X-ray $(0.1-2.4 \mathrm{keV})$ and far-infrared $(\lambda=60-100 \mu \mathrm{m})$ emission from the ROSAT/IRAS sample. Since the CO line widths $\left(W_{\mathrm{CO}}\right)$ reflect the orbital motion in the gravitational potential of the host galaxy, we study how the kinematics are affected by the central massive black hole $(\mathrm{BH})$, using the $\mathrm{X}$-ray luminosity. A significant correlation is found between the $\mathrm{CO}$ line width and hard (0.3-8 keV from Chandra and XMM-Newton) X-ray luminosity for both types of Seyfert nuclei. Assuming an Eddington accretion to estimate the $\mathrm{BH}$ mass $\left(M_{\mathrm{BH}}\right)$ from the X-ray luminosity, the $W_{\mathrm{CO}}-L_{X}$ relation establishes a direct connection between the kinematics of the molecular gas of the host galaxy and the nuclear activity, and corroborates the previous studies that show that the $\mathrm{CO}$ is a good surrogate for the bulge mass. We also find a tight correlation between the (soft and hard) X-ray and the CO luminosities for both Seyfert types. These results indicate a direct relation between the molecular gas (i.e., star formation activity) of the host galaxy and the nuclear activity. To establish a clear causal connection between molecular gas and the fueling of nuclear activity, high-resolution maps $(<100 \mathrm{pc})$ of the $\mathrm{CO}$ emission of our sample will be required and provided in a forthcoming Atacama Large Millimeter Array observation.
\end{abstract}

Key words: galaxies: active - galaxies: ISM - galaxies: nuclei - galaxies: Seyfert

Online-only material: color figures

\section{INTRODUCTION}

Seyfert galaxies are classified into two types based solely on their optical spectrum properties. Type 1 Seyferts (Sy1s) are those with very broad $\mathrm{HI}, \mathrm{He}$ I, and He II emission lines. The forbidden lines [O III], [N II], and [S II], though narrower than the very broad permitted lines, are still broader than the emission lines in most starburst galaxies. Type 2 Seyferts (Sy2s) have permitted and forbidden lines with approximately the same FWHM, similar to the FWHMs of the forbidden lines in Seyfert $1 \mathrm{~s}$, but do not present a broad-line feature (Osterbrock \& Ferland 2000). Evidence now exists that the main differences in the optical spectra are due to Seyfert nuclei being surrounded by a torus of dusty, obscuring gas a few parsecs from the center. In Sy1s the orientation of the torus axis is close to the line of sight allowing direct observations of the inner broader region associated with the accretion disk of the active galactic nucleus (AGN). In type 2 nuclei, the orientation of the torus shields the nucleus from direct view and only the more extended narrow-line clouds are observed. Several studies analyzing the properties of Seyfert nuclei galaxies have reported a difference in the infrared properties between the Seyfert types, where type 2 nuclei present nearly an order of magnitude enhancement of their infrared emission from their disk with respect to those with type 1 nuclei (Edelson et al. 1987; Maiolino et al. 1995). These results can be easily explained in terms of an elevated rate of star formation of massive stars in galaxies with type 2 nuclei. In addition, Maiolino et al. (1997) found, through their ${ }^{12} \mathrm{CO}$ (1-0) observations of a large sample of Seyfert galaxies, no significant difference in the total amount of molecular gas as a function of the Seyfert nuclear type. Therefore, they concluded that the total amount of molecular gas is not responsible for the enhanced star-forming activity in Seyfert 2 hosts. Contrary to those observations, Heckman et al. (1989) and Curran (2000), using observations of the CO $J=1-0$ line, found that Sy2s do indeed have a higher molecular gas content than Seyfert 1s. Curran (2000) found that for Seyfert galaxies with far-infrared (FIR) luminosity $\left(L_{\mathrm{FIR}}\right) \approx 10^{10} L_{\odot}$, the CO/FIR luminosity ratio in type 2 is at least three times that in type 1 sources. They suggested that this molecular gas may be related in some indirect way to the nuclear material hypothesized to obscure the broad-line region in Sy2s. In this paper, we study the molecular gas content of Seyfert types by comparing the luminosities of a higher excitation line (CO $J=2-1)$ to the FIR and $\mathrm{X}$-ray luminosities of a sample of 35 active galaxies. The sources were selected by their soft X-ray (0.1-2.4 keV) and FIR emission from a ROSAT/IRAS sample generated originally by Boller et al. (1992) and modified by Condon et al. (1998), who used new Very Large Array images of all the objects to eliminate uncertainties coming from the original ROSAT and IRAS positions. Molecular gas is important not only to support the star formation activity in the different Seyfert types, but also to understand the powering mechanism of the nuclear activity in AGNs. The source of the accreted gas is still unclear. Most studies suggest that the massive black holes (BHs) are fed by infalling interstellar gas, which is mostly in molecular form in the central kiloparsec of spiral galaxies. Single dish observations of $\mathrm{CO}$ emission cannot give a direct answer to the moleculargas-nuclear-activity relation, since any relation between the fueling (through CO luminosity) and the massive BH data, such as X-ray luminosity (assuming most of the X-ray luminosity in Seyfert galaxies comes from the accretion disk around the massive $\mathrm{BH}$ sitting in the center of the galaxy), must be searched for in the central few $100 \mathrm{pc}$ of nearby AGNs. However, Yamada (1994) and Kawakatu et al. (2005) reveal an interesting close relation between $L_{\mathrm{CO}}$ and $L_{X}$ in Seyfert 1s at low and high redshift, respectively. In this paper, we study the properties of an extended Seyfert galaxy sample including both types of Seyfert 
Table 1

Summary of Galaxies

\begin{tabular}{|c|c|c|c|c|c|}
\hline Galaxy & Name & Type & $\begin{array}{l}\text { Velocity } \\
\left(\mathrm{km} \mathrm{s}^{-1}\right)\end{array}$ & Redshift & $\begin{array}{c}i^{\mathrm{a}} \\
(\mathrm{deg})\end{array}$ \\
\hline IRAS 00076-0459 & MRK 0937 & $\mathrm{SBa} / \mathrm{b}, \mathrm{H}$ II, Sy2 & 8846 & 0.029507 & 29.1 \\
\hline IRAS 00317-2142 & ESO 540- G 001 & $\mathrm{SB}(\mathrm{r} 1) \mathrm{bc}, \mathrm{Sy} 1.8$ & 8048 & 0.026845 & 33 \\
\hline IRAS 04260+6444 & NGC 1569 & IBm;Sbrst, Sy1 & -104 & -0.000347 & 64.7 \\
\hline IRAS $04565+0454$ & UGC 03223 & SBa Sy1 & 4683 & 0.015621 & 61.5 \\
\hline IRAS $05128+5308$ & CGCG 258-006 & S, Sy 1.8 & 8482 & 0.028293 & 68.6 \\
\hline IRAS 05497-0728 & NGC 2110 & SAB0-, Sy2 & 2335 & 0.007789 & 46.4 \\
\hline IRAS 06280+6342 & UGC 03478 & $\mathrm{Sb}, \mathrm{Sy} 1.2$ & 3828 & 0.012769 & 76.7 \\
\hline IRAS $07388+4955$ & UGC 03973 & SBb, Sy1.2 & 6652 & 0.022189 & 36.7 \\
\hline IRAS 08331-0354 & NGC 2617 & Sy1.8 & 4261 & 0.014213 & 39.8 \\
\hline IRAS $09585+5555$ & NGC 3079 & SB(s)c;LINER, Sy2 & 1116 & 0.00372 & 82.5 \\
\hline IRAS $10126+7339$ & NGC 3147 & SA(rs)bc;H II, Sy2 & 2820 & 0.009407 & 29.5 \\
\hline IRAS 10291+6517 & NGC 3259 & SAB(rs)bc:BLAGN, Sy1 & 1686 & 0.005624 & 60.7 \\
\hline IRAS 10295-1831 & 2MASX J10315733-1846333 & $\mathrm{Sp}, \mathrm{Sy} 1$ & 12070 & 0.040261 & 20.3 \\
\hline IRAS 10589-1210 & MCG -02-28-039 & Sy1.5 & 7720 & 0.025751 & 59.9 \\
\hline IRAS $11033+7250$ & NGC 3516 & (R)SB(s): Sy 1.5 & 2649 & 0.008836 & 37.0 \\
\hline IRAS 11083-2813 & ESO 438- G 009 & RSB(rl)ab Sy1.5 & 7199 & 0.024013 & 50.8 \\
\hline IRAS 11112+0951 & IC 2637 & E+, pec, Sy1.5 & 8763 & 0.029230 & 35 \\
\hline IRAS 11210-0823 & NGC 3660 & SB(r)bc Sy2 & 3683 & 0.012285 & 32.9 \\
\hline IRAS $11376+2458$ & NGC 3798 & SB0, Sy1 & 3552 & 0.011848 & 90 \\
\hline IRAS $11395+1033$ & NGC 3822 & Sb Sy2 & 6138 & 0.020474 & 76.0 \\
\hline IRAS 11500-0455 & MCG -01-30-041 & (R)SB(rs)ab, Sy1.8 & 5641 & 0.018816 & 55.5 \\
\hline IRAS $12373-1120$ & MESSIER 104 & SA(s)a;LINER, Sy1.9 & 1024 & 0.003416 & 78.5 \\
\hline IRAS $12393+3520$ & NGC 4619 & SB(r)b pec? Sy1 & 6927 & 0.023106 & 14.6 \\
\hline IRAS $12409+7823$ & 2MASX J12423600+7807203 & Sy1.9 & 6625 & 0.022100 & 75.7 \\
\hline IRAS 12495-1308 & NGC 4748 & Sa Sy 1 & 4386 & 0.014630 & 53.2 \\
\hline IRAS 13218-1929 & 2MASX J13243528-1945114 & $\left(R_{1}^{\prime}\right) \mathrm{SB}(1:) \mathrm{a}, \mathrm{Sy} 1.9$ & 5284 & 0.017625 & $\ldots$ \\
\hline IRAS $14060+7207$ & NGC 5643 & Sy 1.9 & 10251 & 0.034194 & 54.2 \\
\hline IRAS $14105+3932$ & NGC 5515 & Sab, Sy1.9 & 7719 & 0.025749 & 62.6 \\
\hline IRAS $14156+2522$ & NGC 5548 & $\left(\mathrm{R}^{\prime}\right) \mathrm{SA}(\mathrm{s}) 0 / \mathrm{a}$ Sy 1.5 & 5149 & 0.017175 & 41.4 \\
\hline IRAS 14294-4357 & NGC 5643 & SAB(rs)c, Sy2 & 1199 & 0.003999 & 30.5 \\
\hline IRAS 15361-0313 & CGCG 022-021 & H II, Sy1.9 & 7137 & 0.023806 & 68.7 \\
\hline IRAS 15564+6359 & CGCG 319-034 & Sy1.9 & 9023 & 0.030097 & 54.2 \\
\hline IRAS $16277+2433$ & VV 807 & Sy 1.9 & 11241 & 0.037496 & 57.2 \\
\hline IRAS $17020+4544$ & B3 $1702+457$ & SBab;Sy1,Sy2 & 18107 & 0.060400 & 59.9 \\
\hline IRAS 17023-0128 & UGC 10683 & S0+ pec: Sy1.5 & 9149 & 0.030518 & 41.3 \\
\hline IRAS $18001+6638$ & NGC 6552 & SB?, Sy2 & 7942 & 0.026492 & 47.4 \\
\hline IRAS 19399-1026 & NGC 6814 & SAB(rs)bc;H II, Sy1.5 & 1563 & 0.005214 & 85.6 \\
\hline IRAS 20069+5929 & CGCG 303-017 & Sbrst, Sy2 & 11132 & 0.037132 & 48.8 \\
\hline IRAS $20437+5929$ & MRK 0896 & $\mathrm{SBb}, \mathrm{Sy} 1$ & 7922 & 0.026424 & 64.0 \\
\hline IRAS 22062-2803 & NGC 7214 & SB(s)bc pec: Sy1.2 & 6934 & 0.023128 & 47.3 \\
\hline IRAS 22330-2618 & NGC 7314 & SAB(rs)bc, Sy1.9 & 1428 & 0.004763 & 70.3 \\
\hline IRAS 22595+1541 & NGC 7465 & $\left(R^{\prime}\right) \mathrm{SB}(\mathrm{s}): \mathrm{Sy} 2$ & 1968 & 0.006565 & 63.4 \\
\hline IRAS $23111+1344$ & NGC 7525 & E, Sy 1.5 & 12262 & 0.040900 & 30.6 \\
\hline IRAS 23163-0001 & NGC 7603 & SA(rs)b: pec, Sy1.5 & 8851 & 0.029524 & 61.5 \\
\hline IRAS 23279-0244 & UM 163 & SB(r)b pec:;H II, Sy1 & 10022 & 0.033430 & 65.1 \\
\hline IRAS 23566-0424 & IC 1490 & SA(rs)b pec:, Sy1 & 5744 & 0.019160 & 55.8 \\
\hline
\end{tabular}

Note.

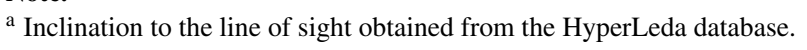

galaxies, from the kinematics and multi-wavelength luminosity comparison point of view. In Section 2, observations and results in terms of line intensities, correlation coefficients, and linear fits are presented. In Section 3, we summarize the results and discuss the interpretation of the $\mathrm{CO}$ line width $\left(W_{\mathrm{CO}}\right)-L_{X}, L_{\mathrm{CO}}-L_{\mathrm{FIR}}$ and $L_{\mathrm{CO}}-L_{X}$ relations.

\section{OBSERVATIONS AND RESULTS}

We have used the $10.4 \mathrm{~m}$ Caltech Submillimeter Observatory (CSO) telescope to observe the CO $J=2-1$ transition line toward the center of 45 Seyfert galaxies and have detected 35 of them. Table 1 lists the source parameters obtained from the NASA/IPAC Extragalactic Database ${ }^{1}$ and the HyperLeda database. ${ }^{2}$ The observations were mostly done during the winter and spring of 2009, with system noise temperatures typically $300-400 \mathrm{~K}$. Pointing was checked regularly on planets with typical pointing errors less than $5^{\prime \prime}$ during one night. The CSO beam size for the ${ }^{12} \mathrm{CO} J=2-1$ line (with rest frequency ${ }^{3}$ equal to $230.538 \mathrm{GHz}$ ) is approximately $30^{\prime \prime}$ and the beam efficiency is 0.69. Peak intensities in $T_{A}^{*}$ scale are shown in Table 2. $T_{A}^{*}$ is

\footnotetext{
See http://nedwww.ipac.caltech.edu/

See http://leda.univ-lyon1.fr/

3 See http://physics.nist.gov/cgi-bin/micro/table5/start.pl
} 
Table 2

Observational Parameters and Gaussian Line Fits

\begin{tabular}{|c|c|c|c|c|c|c|c|c|c|}
\hline Source & $\begin{array}{l}\operatorname{Size}^{\mathrm{a}} \\
(\mathrm{kpc})\end{array}$ & $\begin{array}{c}\Delta V^{\mathrm{b}} \\
\left(\mathrm{km} \mathrm{s}^{-1}\right)\end{array}$ & $\begin{array}{c}I^{\mathrm{c}}=\int T_{A}^{*} d V \\
\left(\mathrm{~K} \mathrm{~km} \mathrm{~s}^{-1}\right)\end{array}$ & $\begin{array}{c}\text { Intensity }^{\mathrm{d}} \\
\left(\mathrm{erg} \mathrm{cm} \mathrm{cm}^{-2} \mathrm{~s}^{-1} \mathrm{sr}^{-1}\right)\end{array}$ & $\begin{array}{c}S_{\mathrm{CO}}{ }^{\mathrm{e}} \\
\left(\mathrm{Jy} \mathrm{km} \mathrm{s}^{-1}\right)\end{array}$ & $\begin{array}{c}\log L_{\mathrm{CO}}{ }^{\mathrm{f}} \\
\left(\mathrm{K} \mathrm{km} \mathrm{s}^{-1} \mathrm{pc}^{2}\right)\end{array}$ & $\begin{array}{l}\log L_{\mathrm{FIR}}{ }^{\mathrm{g}} \\
\quad\left(L_{\odot}\right)\end{array}$ & $\begin{array}{l}\log L_{x}{ }^{\mathrm{h}} \\
\left(\operatorname{erg~s}^{-1}\right)\end{array}$ & $\begin{array}{c}S_{X}^{\mathrm{i}} \\
\left(\mathrm{erg} \mathrm{s}^{-1} \mathrm{~cm}^{-2}\right)\end{array}$ \\
\hline IRAS 00076-0459 & 16.71 & $100 \pm 20$ & $1.18 \pm 0.2$ & $2.15 \times 10^{-8} \pm 3.6 \times 10^{-9}$ & $73.1 \pm 12$ & $8.77 \pm 0.0059$ & 10.18 & 42.94 & \\
\hline IRAS 00317-2142 & 15.20 & $150 \pm 15$ & $5.45 \pm 0.9$ & $9.93 \times 10^{-8} \pm 1.6 \times 10^{-8}$ & $335.01 \pm 54$ & $9.35 \pm 0.0068$ & 10.94 & 42.81 & $7.49 \times 10^{-13}$ \\
\hline IRAS 04260+6444 & 0.19 & $50 \pm 7$ & $1.01 \pm 0.18$ & $1.85 \times 10^{-8} \pm 3.3 \times 10^{-9}$ & $57.69 \pm 10$ & $4.8 \pm 0.075$ & $\ldots$ & 37.92 & $1.62 \times 10^{-13}$ \\
\hline IRAS $04565+0454$ & 8.85 & $400 \pm 50$ & $1.68 \pm 0.2$ & $3.06 \times 10^{-8} \pm 3.6 \times 10^{-9}$ & $99.82 \pm 12$ & $8.35 \pm 0.013$ & 10.06 & 42.06 & $\ldots$ \\
\hline IRAS $05128+5308$ & 16.02 & $560 \pm 60$ & $4.09 \pm 0.7$ & $7.46 \times 10^{-8} \pm 1.3 \times 10^{-8}$ & $252.84 \pm 44$ & $9.28 \pm 0.0067$ & 10.81 & 42.06 & $\ldots$ \\
\hline IRAS 05497-0728 & 4.41 & $\ldots$ & $\leqslant 1.08$ & $\ldots$ & $\ldots$ & $\leqslant 7.55$ & 9.72 & 40.99 & $1.04 \times 10^{-11}$ \\
\hline IRAS $06280+6342$ & 7.23 & $270 \pm 50$ & $1.79 \pm 0.17$ & $3.26 \times 10^{-8} \pm 3.1 \times 10^{-9}$ & $105.46 \pm 10$ & $8.21 \pm 0.013$ & 9.84 & 42.61 & $\ldots$ \\
\hline IRAS $07388+4955$ & 12.56 & $450 \pm 25$ & $1.21 \pm 0.16$ & $2.19 \times 10^{-8} \pm 2.9 \times 10^{-9}$ & $73.06 \pm 9.7$ & $8.52 \pm 0.0079$ & 10.31 & 43.84 & $3.18 \times 10^{-11}$ \\
\hline IRAS 08331-0354 & 8.05 & $180 \pm 30$ & $2.22 \pm 0.65$ & $4.04 \times 10^{-8} \pm 1.2 \times 10^{-8}$ & $131.4 \pm 39$ & $8.39 \pm 0.036$ & 9.73 & 42.27 & $\ldots$ \\
\hline IRAS $09585+5555$ & 2.11 & $400 \pm 120$ & $3.21 \pm 0.3$ & $5.84 \times 10^{-8} \pm 5.4 \times 10^{-9}$ & $184.15 \pm 17$ & $7.37 \pm 0.034$ & 10.36 & 40.18 & $6.39 \times 10^{-13}$ \\
\hline IRAS $10126+7339$ & 5.33 & $430 \pm 30$ & $9.33 \pm 1.1$ & $1.70 \times 10^{-7} \pm 2.0 \times 10^{-8}$ & $544.49 \pm 64$ & $8.65 \pm 0.024$ & 10.51 & 41.83 & $1.05 \times 10^{-12}$ \\
\hline IRAS $10291+6517$ & 3.18 & $500 \pm 40$ & $4.28 \pm 0.8$ & $7.81 \times 10^{-8} \pm 1.5 \times 10^{-8}$ & $247.45 \pm 48$ & $7.86 \pm 0.083$ & 9.07 & 40.04 & $3.31 \times 10^{-14}$ \\
\hline IRAS 10295-1831 & 22.80 & $150 \pm 12$ & $1.45 \pm 0.15$ & $2.64 \times 10^{-8} \pm 2.7 \times 10^{-9}$ & $92.88 \pm 9.5$ & $9.08 \pm 0.002$ & 11.02 & 43.14 & $\ldots$ \\
\hline IRAS 10589-1210 & 14.58 & $510 \pm 30$ & $1.86 \pm 0.2$ & $3.40 \times 10^{-8} \pm 3.6 \times 10^{-9}$ & $114.40 \pm 12$ & $8.85 \pm 0.0048$ & 10.55 & 41.93 & $\ldots$ \\
\hline IRAS $11033+7250$ & 5.00 & & $\leqslant 1.53$ & & & $\leqslant 7.81$ & 9.55 & 41.73 & $6.21 \times 10^{-11}$ \\
\hline IRAS 11083-2813 & 13.59 & $300 \pm 50$ & $1.95 \pm 0.3$ & $3.56 \times 10^{-8} \pm 5.5 \times 10^{-9}$ & $119.13 \pm 18$ & $8.81 \pm 0.0078$ & 10.66 & 42.95 & $\ldots$ \\
\hline IRAS $11112+0951$ & 16.55 & $700 \pm 50$ & $6.34 \pm 0.9$ & $1.15 \times 10^{-7} \pm 1.6 \times 10^{-8}$ & $391.90 \pm 54$ & $9.49 \pm 0.005$ & 10.65 & 42.91 & $\ldots$ \\
\hline IRAS $11210-0823$ & 6.96 & $450 \pm 30$ & $2.57 \pm 0.28$ & $4.68 \times 10^{-7} \pm 5.1 \times 10^{-9}$ & $151.25 \pm 65$ & $8.33 \pm 0.063$ & 9.92 & 41.61 & $7.60 \times 10^{-12}$ \\
\hline IRAS $11376+2458$ & 6.71 & $400 \pm 50$ & $1.63 \pm 0.3$ & $2.97 \times 10^{-8} \pm 5.5 \times 10^{-9}$ & $95.84 \pm 18$ & $8.09 \pm 0.029$ & 9.64 & 41.46 & 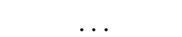 \\
\hline IRAS $11395+1033$ & 11.59 & $700 \pm 200$ & $6.24 \pm 0.6$ & $1.14 \times 10^{-7} \pm 1.1 \times 10^{-8}$ & $376.55 \pm 36$ & $9.17 \pm 0.0065$ & 10.58 & 41.91 & $\ldots$ \\
\hline IRAS 11500-0455 & 10.65 & $400 \pm 50$ & $1.16 \pm 0.16$ & $2.12 \times 10^{-8} \pm 2.9 \times 10^{-9}$ & $69.82 \pm 9.6$ & $8.36 \pm 0.011$ & 10.09 & 42.12 & $\ldots$ \\
\hline IRAS $12373-1120$ & 1.93 & $420 \pm 50$ & $0.88 \pm 0.2$ & $1.61 \times 10^{-8} \pm 2.2 \times 10^{-9}$ & $50.89 \pm 5.0$ & $6.73 \pm 0.042$ & 9.35 & 40.75 & $1.61 \times 10^{-12}$ \\
\hline IRAS $12393+3520$ & 13.08 & $210 \pm 50$ & $2.52 \pm 0.23$ & $4.60 \times 10^{-8} \pm 4.2 \times 10^{-9}$ & $153.57 \pm 14$ & $8.88 \pm 0.005$ & 10.09 & 42.12 & $\ldots$ \\
\hline IRAS $12409+7823$ & 12.51 & $\ldots$ & $\leqslant 0.86$ & & $\ldots$ & $\leqslant 8.38$ & 9.94 & 41.87 & $\ldots$ \\
\hline IRAS $12495-1308$ & 8.28 & $300 \pm 150$ & $3.24 \pm 0.4$ & $5.91 \times 10^{-8} \pm 7.3 \times 10^{-9}$ & $192.42 \pm 24$ & $8.58 \pm 0.014$ & 9.83 & 42.06 & $\ldots$ \\
\hline IRAS 13218-1929 & 9.98 & $360 \pm 30$ & $5.01 \pm 0.49$ & $9.11 \times 10^{-8} \pm 8.9 \times 10^{-9}$ & $299.28 \pm 29$ & $8.93 \pm 0.0084$ & 10.29 & 42.14 & $\ldots$ \\
\hline IRAS $14060+7207$ & 19.36 & $\ldots$ & $\leqslant 1.33$ & & & $\leqslant 8.96$ & 10.26 & 42.15 & $\ldots$ \\
\hline IRAS $14105+3932$ & 14.58 & $650 \pm 50$ & $4.83 \pm 0.66$ & $8.81 \times 10^{-8} \pm 1.2 \times 10^{-8}$ & $296.26 \pm 40$ & $9.26 \pm 0.0062$ & 10.63 & 41.65 & $\ldots$ \\
\hline IRAS $14156+2522$ & 9.73 & $400 \pm 100$ & $2.72 \pm 0.66$ & $4.96 \times 10^{-8} \pm 1.2 \times 10^{-8}$ & $162.68 \pm 39$ & $8.65 \pm 0.0022$ & 9.93 & 43.67 & $5.39 \times 10^{-11}$ \\
\hline IRAS 14294-4357 & 2.26 & $180 \pm 15$ & $15.88 \pm 1.04$ & $2.89 \times 10^{-7} \pm 1.9 \times 10^{-8}$ & $911.72 \pm 60$ & $8.13 \pm 0.024$ & 9.85 & 40.61 & $5.31 \times 10^{-13}$ \\
\hline IRAS $15361-0313$ & 13.48 & $400 \pm 50$ & $6.40 \pm 1.17$ & $1.17 \times 10^{-7} \pm 2.1 \times 10^{-8}$ & $389.84 \pm 70$ & $9.31 \pm 0.0094$ & 10.79 & 42.02 & $\ldots$ \\
\hline IRAS $15564+6359$ & 17.04 & $600 \pm 60$ & $2.90 \pm 0.67$ & $5.29 \times 10^{-7} \pm 1.2 \times 10^{-8}$ & $180.10 \pm 41$ & $9.18 \pm 0.0078$ & 10.41 & 42.46 & $1.12 \times 10^{-12}$ \\
\hline IRAS $16277+2433$ & 21.23 & $\ldots$ & $\leqslant 1.45$ & $\ldots$ & $\ldots$ & $\leqslant 9.08$ & 10.53 & 42.02 & $2.25 \times 10^{-12}$ \\
\hline IRAS $17020+4544$ & 34.20 & $\ldots$ & $\leqslant 1.11$ & $\ldots$ & & $\leqslant 9.40$ & 11.02 & 44.06 & $1.30 \times 10^{-11}$ \\
\hline IRAS $17023-0128$ & 17.28 & $\ldots$ & $\leqslant 0.76$ & $\ldots$ & $\ldots$ & $\leqslant 8.62$ & 10.42 & 42.95 & $\ldots$ \\
\hline IRAS $18001+6638$ & 15.00 & $500 \pm 100$ & $1.72 \pm 0.5$ & $3.14 \times 10^{-8} \pm 9.1 \times 10^{-9}$ & $105.99 \pm 31$ & $8.84 \pm 0.013$ & 10.63 & $\ldots$ & $2.94 \times 10^{-13}$ \\
\hline IRAS 19399-1026 & 2.95 & $140 \pm 20$ & $4.36 \pm 0.68$ & $7.95 \times 10^{-8} \pm 1.2 \times 10^{-8}$ & $251.69 \pm 38$ & $7.80 \pm 0.048$ & 9.81 & 41.91 & $3.08 \times 10^{-11}$ \\
\hline IRAS 20437-0259 & 14.96 & $\ldots$ & $\leqslant 0.97$ & & $\ldots$ & $\leqslant 8.61$ & 10.04 & 43.31 & $1.06 \times 10^{-11}$ \\
\hline IRAS 22062-2803 & 13.09 & $500 \pm 100$ & $6.97 \pm 0.7$ & $1.27 \times 10^{-7} \pm 1.3 \times 10^{-8}$ & $423.65 \pm 43$ & $9.33 \pm 0.0056$ & 10.56 & 43.04 & $\ldots$ \\
\hline IRAS 22330-2618 & 2.69 & $140 \pm 50$ & $1.91 \pm 0.3$ & $3.47 \times 10^{-8} \pm 5.5 \times 10^{-9}$ & $109.76 \pm 17$ & $7.37 \pm 0.052$ & 9.56 & 41.17 & $1.40 \times 10^{-11}$ \\
\hline IRAS $22595+1541$ & 3.72 & $190 \pm 30$ & $3.07 \pm 0.6$ & $5.59 \times 10^{-8} \pm 1.1 \times 10^{-8}$ & $177.79 \pm 35$ & $7.85 \pm 0.055$ & 9.86 & 40.54 & $1.54 \times 10^{-12}$ \\
\hline IRAS $23111+1344$ & 23.16 & $\ldots$ & $\leqslant 0.65$ & $\ldots$ & $\ldots$ & $\leqslant 8.83$ & 10.62 & 42.81 & $\ldots$ \\
\hline IRAS 23163-0001 & 16.71 & $550 \pm 100$ & $3.22 \pm 0.4$ & $5.86 \times 10^{-8} \pm 7.3 \times 10^{-9}$ & $199.27 \pm 25$ & $9.21 \pm 0.0045$ & 10.41 & 43.3 & $3.65 \times 10^{-11}$ \\
\hline IRAS 23279-0244 & 18.93 & $610 \pm 50$ & $4.75 \pm 0.6$ & $8.64 \times 10^{-8} \pm 1.1 \times 10^{-8}$ & $297.36 \pm 38$ & $9.49 \pm 0.0036$ & 10.35 & 42.64 & $\ldots$ \\
\hline IRAS 23566-0424 & 10.85 & $470 \pm 50$ & $3.35 \pm 0.6$ & $6.09 \times 10^{-8} \pm 1.1 \times 10^{-8}$ & $201.05 \pm 36$ & $8.83 \pm 0.014$ & 10.01 & 42.83 & $\ldots$ \\
\hline
\end{tabular}

Notes.

a Beam size at each source.

b Width of the line at $20 \%$ of the peak intensity.

${ }^{\mathrm{c}}$ Integrated intensity in $T_{A}^{*}$ corrected for beam efficiency. For the non-detection an upper limit of $3 \sigma$ has been used.

${ }^{\mathrm{d}}$ Intensity from the Rayleigh-Jeans relations.

e Flux.

${ }^{\mathrm{f}}$ CO luminosity, see the Appendix for derivation.

$\mathrm{g}$ FIR luminosity in the wavelength range $40 \mu \mathrm{m} \leqslant \lambda \leqslant 120 \mu \mathrm{m}$.

h Soft (0.1-2.4 keV) X-ray luminosity from ROSAT data.

${ }^{\mathrm{i}}$ Hard absorption-corrected X-ray flux $(0.3-8 \mathrm{keV})$ from Chandra and XMM-Newton data.

converted to the main beam brightness scale, $T_{\mathrm{mb}}$, by dividing the $T_{A}^{*}$ by the beam efficiency. We have applied the necessary corrections, cold spillover and beam coupling, to those sources with size similar to or smaller than the beam. This gives $T_{A}^{*} / \beta$, where $\beta$ is the spillover and beam coupling coefficient. Source sizes have been determined by their optical extent. Since most of our sources are bigger than the beam, we assume extended sources, i.e., $\theta_{\text {beam }} \leqslant \theta_{\text {source. }}$ Therefore, no beam dilution correction has been applied in our temperature calculations. We used a $1 \mathrm{GHz}$ bandwidth FFTS back end with 8192 channels and a $1 \mathrm{GHz}$ bandwidth acousto-optical spectrometer with 2048 channels. 

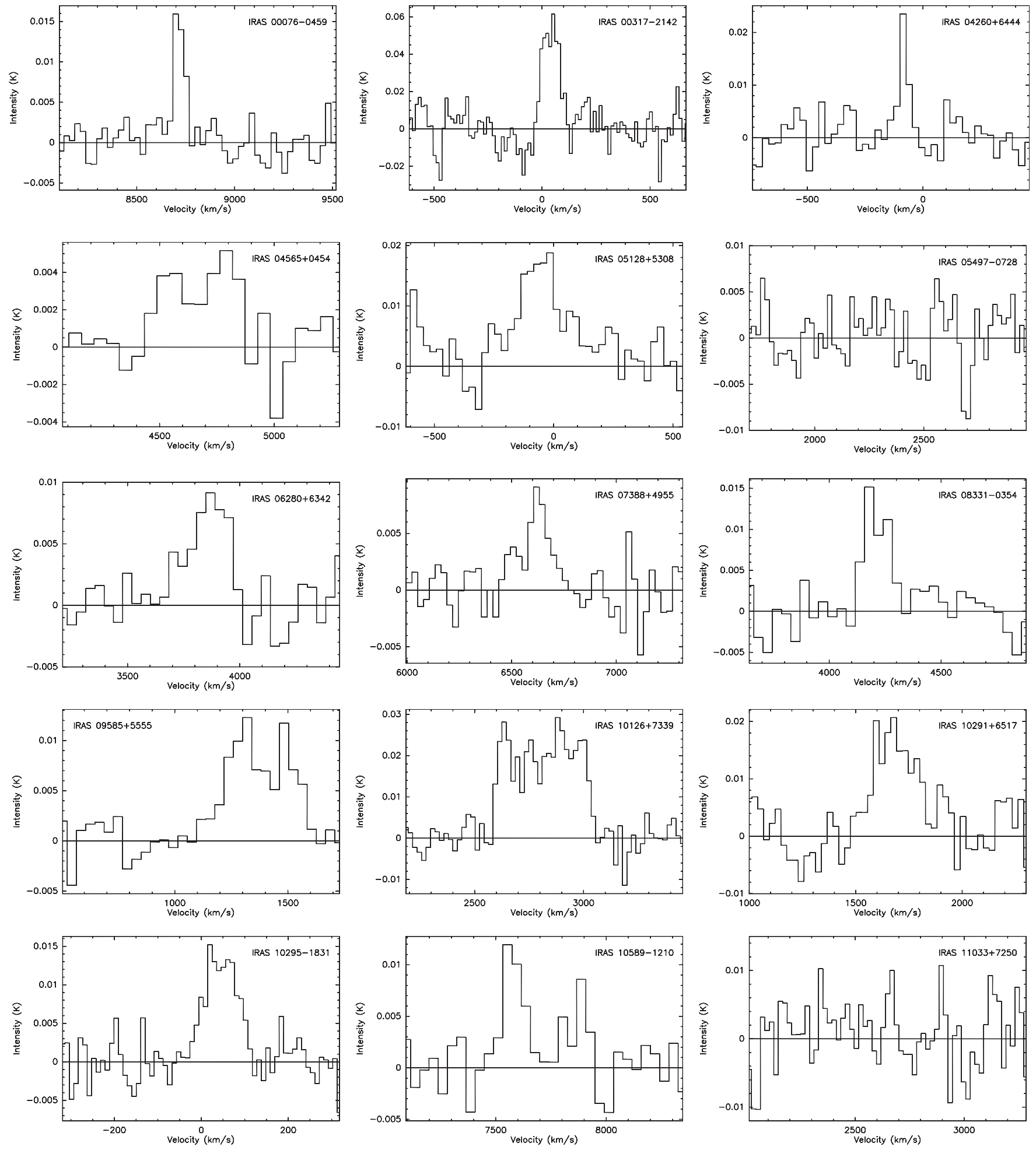

Figure 1. CO $J=2-1$ observations of our sample. The intensity scale is $T_{A}^{*}$ in $\mathrm{K}$.

The molecular line emissions observed for our sample are shown in Figure 1. A first-order polynomial is used to correct the baseline. A $3 \sigma$ detection has been assumed to calculate the upper limit for the non-detected sources.

\subsection{CO Line Width and the Galaxy Inclination}

Figure 2 shows that the $\mathrm{CO}$ line widths correlate with the galaxy inclination. This result shows that the sources have been correctly identified by Condon et al. (1998) in spite of the large pointing errors of IRAS and ROSAT. The line widths were measured at $20 \%$ of the peak intensity since it gives a more robust measurement of the maximum rotation velocity of the disk as shown by Ho (2007). The galactic disk inclination angles of these Seyfert galaxies were estimated from the HyperLeda database. The $\mathrm{CO}$ line width has been also corrected by the blue luminosity $\left(W_{\mathrm{CO}} / L^{1 / 4}\right)$ to avoid selection effects, since brighter sources rotate faster on average (Tully \& Fisher 1977, who 

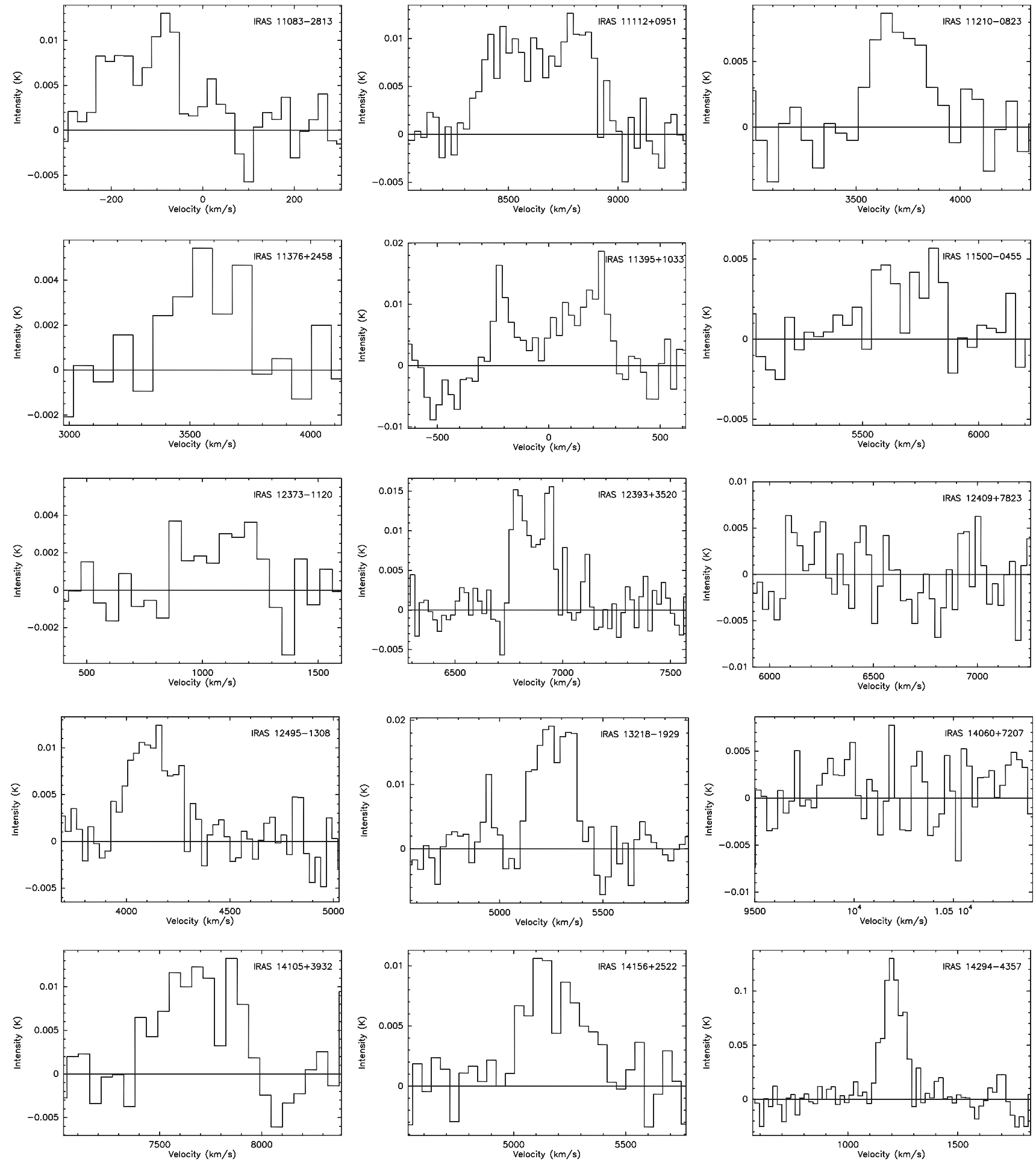

Figure 1. (Continued)

establish that the luminosity, $L$, is proportional to the fourth power of the rotational velocity, $V$, i.e., $L \approx V^{4}$ ). We notice that when applying the luminosity correction the correlation improves slightly and reduces the spread.

Figure 2 shows that face-on galaxies with smaller inclinations tend to have narrower velocities than edge-on galaxies with larger inclinations, following $\Delta V_{\mathrm{CO}} \approx 2 V_{\mathrm{MAX}} \times \sin (i)$. This is an unsurprising result since the $\mathrm{CSO}$ beam samples $\mathrm{CO}$ emission on scales of few kiloparsecs (see Table 2). As molecular disks are roughly coplanar with stellar disks on these scales, a rough correlation is expected.

The inclination-corrected $\mathrm{CO}$ line-width-redshift correlation has been checked for selection effects of more distant objects being more luminous. In our sample the inclinationcorrected CO line-width values are distributed around an average value of $500 \mathrm{~km} \mathrm{~s}^{-1}$ for any redshift, which corresponds to a typical rotational velocity value for spiral galaxies. 

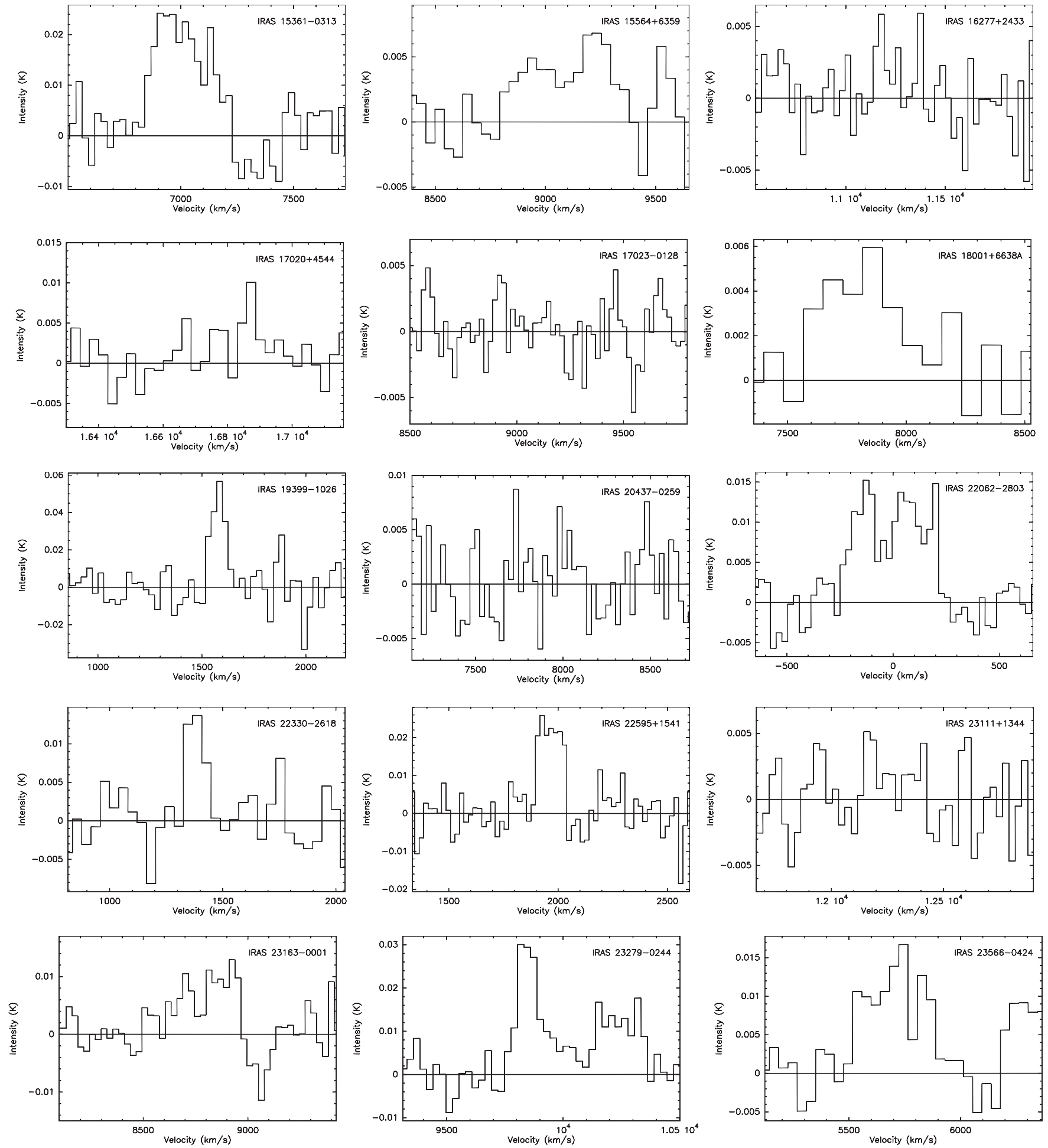

Figure 1. (Continued)

\subsection{CO Line Width and the X-Ray Luminosity}

We run a simple statistical approach in our investigation of the CO kinematics. For all the Seyfert galaxies with detected $\mathrm{CO}$ emission, we examine the possible correlation between the inclination-corrected $\mathrm{CO}$ line width and the soft (ROSAT, 0.1-2.4 keV) and hard (Chandra or XMM-Newton, 0.3-8 keV) X-ray luminosity: the data are listed in Table 2. The hard $\mathrm{X}$-ray luminosities in Table 2 are corrected for absorption using the X-ray fitting software from HEASARC. ${ }^{4}$ For those type 1 (unobscured) AGNs with no available hard X-ray data, we extrapolate the ROSAT $(0.5-2 \mathrm{keV})$ into the Chandra (0.3-8 keV) X-ray fluxes, by using the tool PIMMS,${ }^{5}$ applying the net count rate, a power-law spectrum with index $\Gamma=1.6$, and an estimate of the Galactic neutral hydrogen column density

$\begin{array}{ll}4 & \text { See http://heasarc.gsfc.nasa.gov } \\ 5 & \text { See http://heasarc.gsfc.nasa.gov/Tools/w3pimms.html }\end{array}$ 


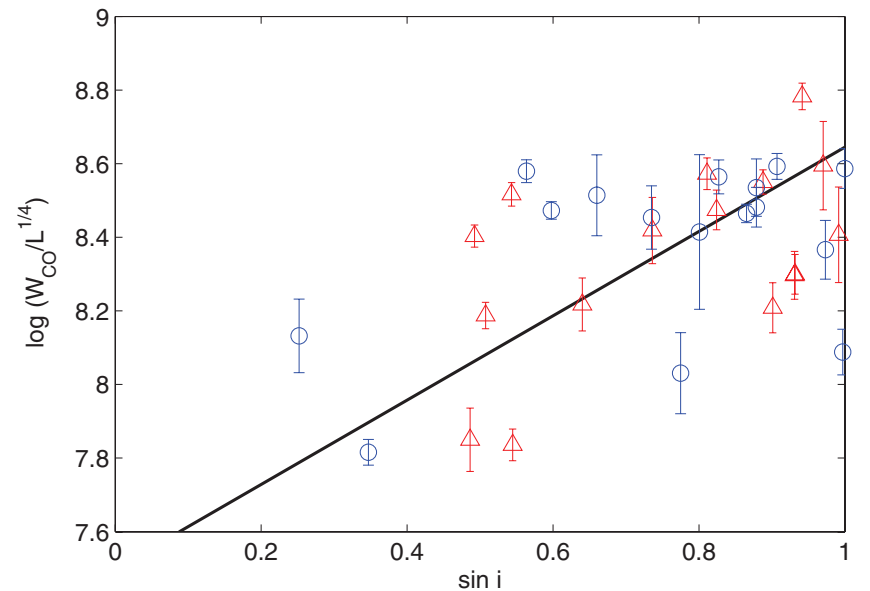

Figure 2. CO line width $\left(\mathrm{km} \mathrm{s}^{-1}\right)$ blue luminosity corrected vs. the sine of the galaxy inclination for the observed sample, Sy1s (circles) and Sy2s (triangles). The CO line width and $\sin i$ are correlated with a 0.49 factor and a 0.21 of null probability (see Table 3 ). The straight line represents the least-squares fit applied to the whole sample (both Sy1s and Sy2s).

(A color version of this figure is available in the online journal.)

from Kalberla et al. (2005). The correlation between X-ray power and $\mathrm{CO}$ kinematics has not been examined before, to the best of the authors' knowledge. Figure 3 shows the soft (0.1-2.4 keV; left) and hard (0.3-8 keV; right) X-ray luminosity, $L_{X}$, versus the corrected CO line width, $W_{\mathrm{CO}}$, in a log-log plot. For the least-squares fit and slope calculations the bisector of the ordinary least-squares regression described in Isobe et al. (1990) has been used. Table 3 lists the results of the $L_{X}-W_{\mathrm{CO}}$ correlation together with the $\chi^{2}$ value and probabilities. The results from Figure 3 show a significant correlation between the $\mathrm{CO}$ line width and both soft and hard X-ray luminosity for Sy 1 galaxies, with a correlation coefficient of 0.67 and 0.62 and a null probability of 0.12 and 0.32 , respectively. In most Sy2s, the observed $0.1-2.4 \mathrm{keV}$ flux is a mixture of emission from the

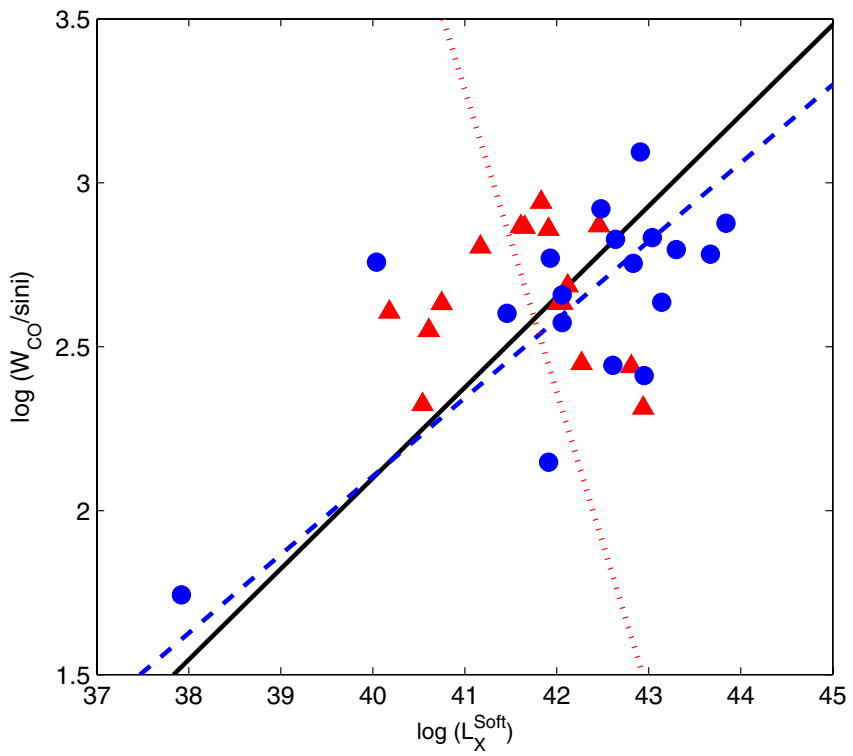

host galaxy, reflected X-ray emission from the AGNs, and, if the absorbing column density is low enough, some transmitted intrinsic flux. The soft $\mathrm{X}$-ray photons, originating within a radius close to the nucleus ( $\leqslant 1 \mathrm{pc}$ ), are absorbed by the dusty torus and only the fraction that is scattered toward our line of sight escapes from above and/or below the torus and can be detected, leading to the reduction in observed X-ray flux from Seyfert $2 \mathrm{~s}$. Consequently, the soft X-ray luminosity is fainter and does not present a significant correlation with the $\mathrm{CO}$ line width.

The observed $\mathrm{CO}$ line width reflects the dynamics of the molecular gas in the inner parts of the galaxy. Therefore, we use the CO line widths to estimate the galaxy dynamical masses in this region. For a rotating disk of radius $R$, the dynamical mass enclosed within $R$ can be determined by the following equation (Solomon et al. 1997):

$$
M_{\mathrm{dyn}} \approx \frac{R \times \Delta V^{2}}{2 \times \sin ^{2}(i) \times G} .
$$

Here, we assume that the gas emission comes from a rotating disk of outer radius $R$ (in units of kiloparsecs) observed at an inclination angle $i$. We use a radius, $R$, equal to the telescope beam FWHM at each source; see Table 2.

At the same time, X-ray luminosity in Seyfert galaxies is related to the black hole mass (BHM; Graham et al. 2001). Given the bolometric luminosity for an AGN, we can estimate the minimum mass of the $\mathrm{BH}$ by using the Eddington limit relation. In some cases the true bolometric luminosity cannot be calculated easily, as in the case of Seyfert $2 \mathrm{~s}$ where the optical and ultraviolet (UV) radiation is highly obscured by the dusty torus. However, we can estimate the BHM from the $\mathrm{X}$-ray luminosity: first, by assuming that an important fraction of the bolometric luminosity is emitted in the X-ray range by the central source; second, the AGN luminosity, $L$, can be estimated from the luminosity in any given band $b, L_{b}$, by applying a suitable bolometric correction $f_{\text {bol }, b}=L / L_{b}$. We will use the bolometric corrections calculated by Marconi et al. (2004).

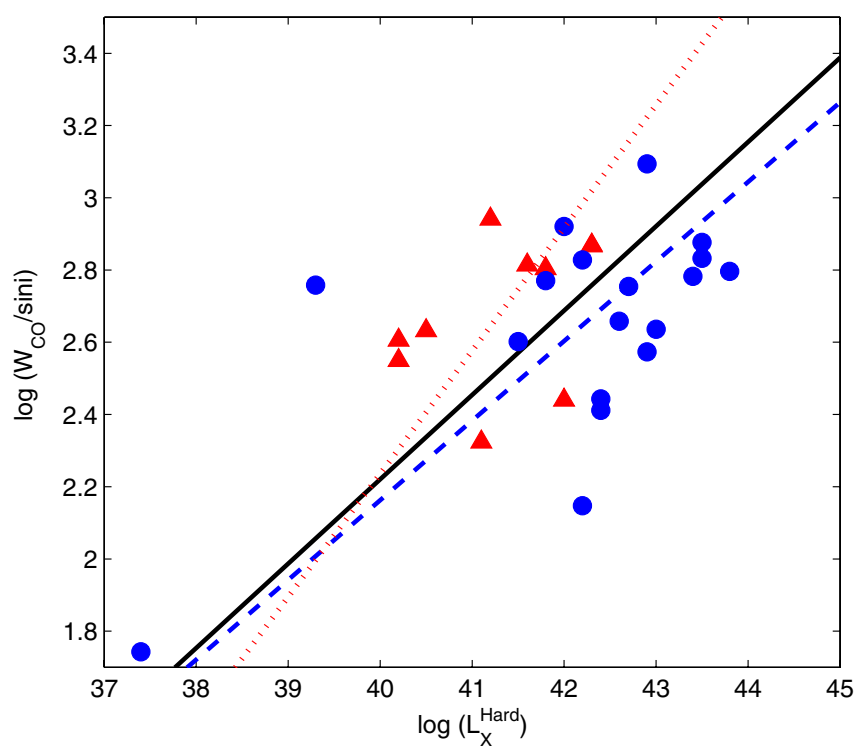

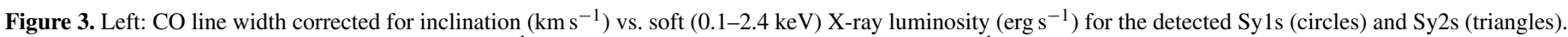

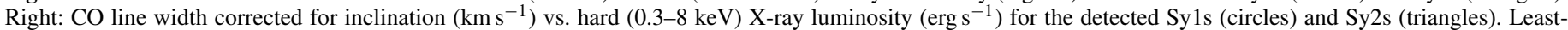

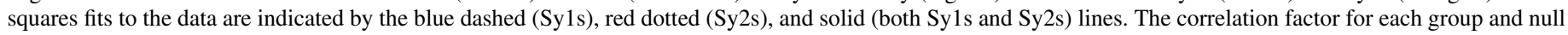
probability are shown in Table 3.

(A color version of this figure is available in the online journal.) 
Table 3

Correlations

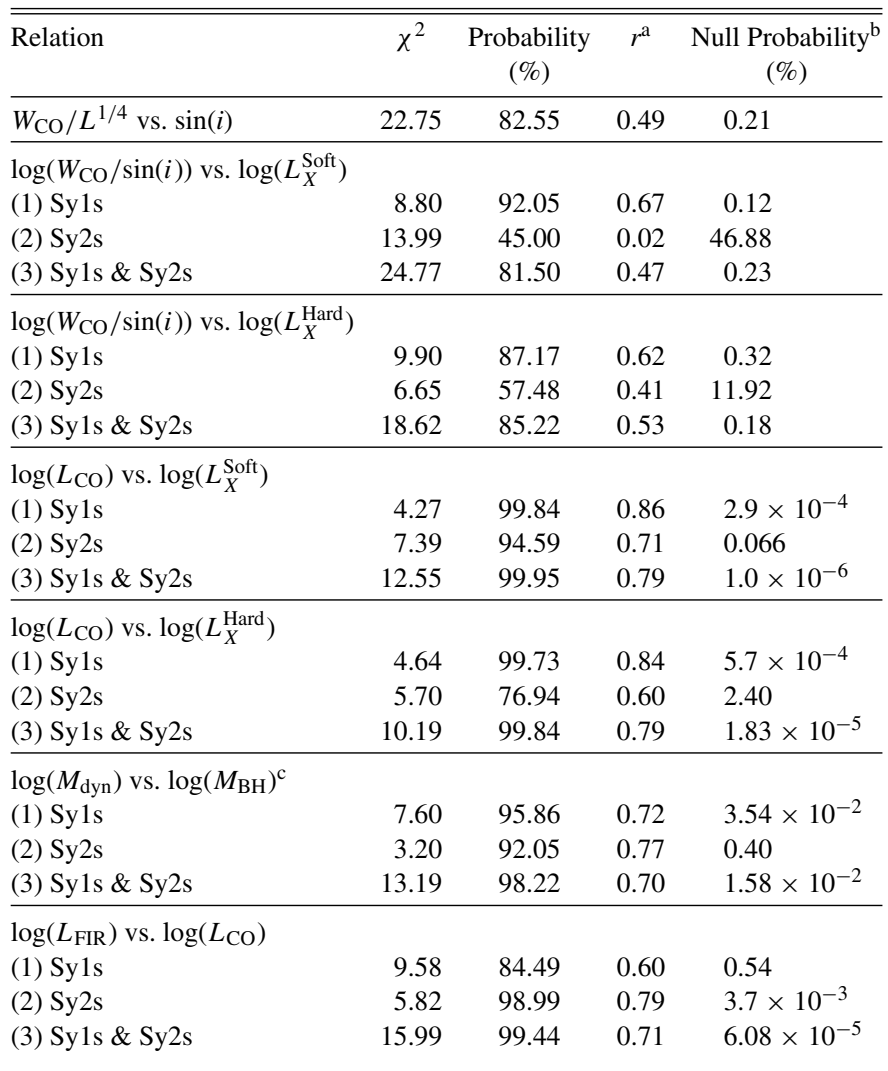

Notes.

a The linear correlation coefficient: $r \equiv \frac{N \sum x_{i} y_{i}-\sum x_{i} \sum y_{i}}{\left.\left[N \sum x_{i}^{2}-\left(\sum x_{i}\right)^{2}\right]^{1 / 2}\left[N \sum y_{i}^{2}-\left(\sum y_{i}\right)^{2}\right]^{1 / 2}\right]}$, where $r$ ranges from 0 , when there is no correlation to 1 , and when there is complete correlation.

b Null probability of the correlation (in percentage); indicates the probability that the observed data could have come from an uncorrelated parent population. A small value of the probability implies that the observed variables are probably correlated.

${ }^{\mathrm{c}}$ Dynamical mass calculated assuming a rotating disk $R$, equal to the telescope beam FWHM at each source.

Marconi et al. (2004) use in their calculations the intrinsic luminosity which is the total luminosity directly produced by the accretion process, i.e., the sum of the optical-UV and X-ray luminosities radiated by the accretion disc and hot corona, respectively. We also assume that the $\mathrm{X}$-ray bolometric correction is the same in Sy1 and Sy2 galaxies. As expected from Figure 3, the dynamical and back hole masses present a significant correlation as shown in Figure 4.

\subsection{Relation between the CO Luminosity with FIR and X-Ray Luminosities}

Figure 5 shows the strong correlation between the FIR luminosity, ${ }^{6} L_{\mathrm{FIR}}$, and the CO line luminosity, $L_{\mathrm{CO}}$, of the Seyfert 1s (circles) and Seyfert 2s (triangles) from our sample, in a $\log -\log$ plot, to confirm the relation observed between far-infrared and CO line luminosity (Rickard \& Harvey 1984; Young et al. 1986; Heckman et al. 1989; Sanders et al. 1991;

\footnotetext{
6 The FIR luminosity, in the wavelength range $40 \mu \mathrm{m}<\lambda<120 \mu \mathrm{m}$ in $\left(\mathrm{W} \mathrm{m}^{-2}\right)$, is estimated using the relation (Helou et al. 1988) $L_{\mathrm{FIR}}=1.26 \times$ $10^{-14}\left(2.58 \times S_{60 \mu \mathrm{m}}+S_{100 \mu \mathrm{m}}\right)$
}

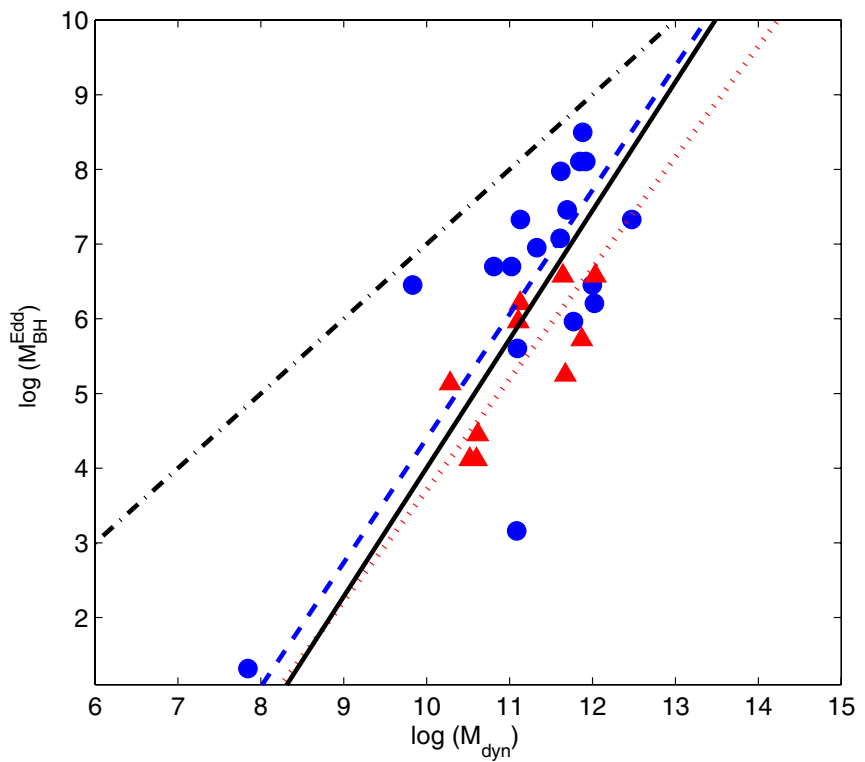

Figure 4. Black hole mass $M_{\mathrm{BH}}^{\mathrm{Edd}}\left[M_{\odot}\right]$ vs. the dynamical mass of the galaxy $M_{\text {dyn }}\left[M_{\odot}\right]$ for both type 1 (filled circles) and type 2 (triangles) Seyfert galaxies. Least-squares fits to the data are indicated by the blue dashed (Sy1s), red dotted (Sy2s), and solid (both Sy1s and Sy2s) lines. The correlation factor for each group and null probability are shown in Table 3 . The $M_{\mathrm{BH}}-M_{\text {bulge }}$ relation for normal galaxies, i.e., $\left\langle M_{\mathrm{BH}} / M_{\text {bulge }}\right\rangle \sim 0.001$, is indicated by the dot-dashed line. (A color version of this figure is available in the online journal.)

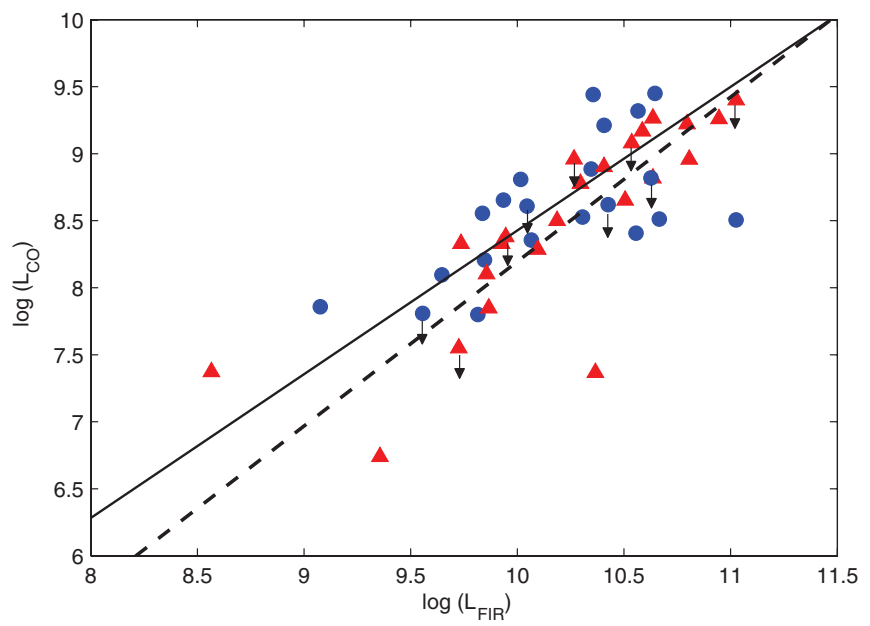

Figure 5. $\log L_{\mathrm{CO}}\left(\mathrm{Kkm} \mathrm{s}^{-1} \mathrm{pc}^{2}\right)$ vs. $\log L_{\mathrm{FIR}}\left[L_{\odot}\right]$ for the observed Sy1s (circles) and Sy2s (triangles). Least-squares fits to the data are indicated by the solid (Sy1s) and dashed (Sy2s) lines. The upper limits of the non-detection are plotted as well. The correlation factor for each group and probability of not being correlated are shown in Table 3 .

(A color version of this figure is available in the online journal.)

Rigopoulou et al. 1996). The strong correlation is interpreted as the result of the link between the amount of molecular gas and the rate of star formation. The FIR emission for both types of galaxies is very similar with a mean ratio of $L_{\mathrm{FIR}}(\mathrm{Sy} 1) / L_{\mathrm{FIR}}(\mathrm{Sy} 2)$ $\approx 0.88$. This result suggests two different possibilities: first, that the FIR emission comes from dust re-radiation of starlight from regions outside the torus, where both classes of Seyferts have similar properties; and second, the FIR in Seyfert galaxies can be powered by the AGN, i.e., non-thermal radiation coming from the nucleus torus, but in that case, the torus should have a similar covering fraction and optical depth to obtain the same emission from Seyfert $2 \mathrm{~s}$ as in $1 \mathrm{~s}$. 

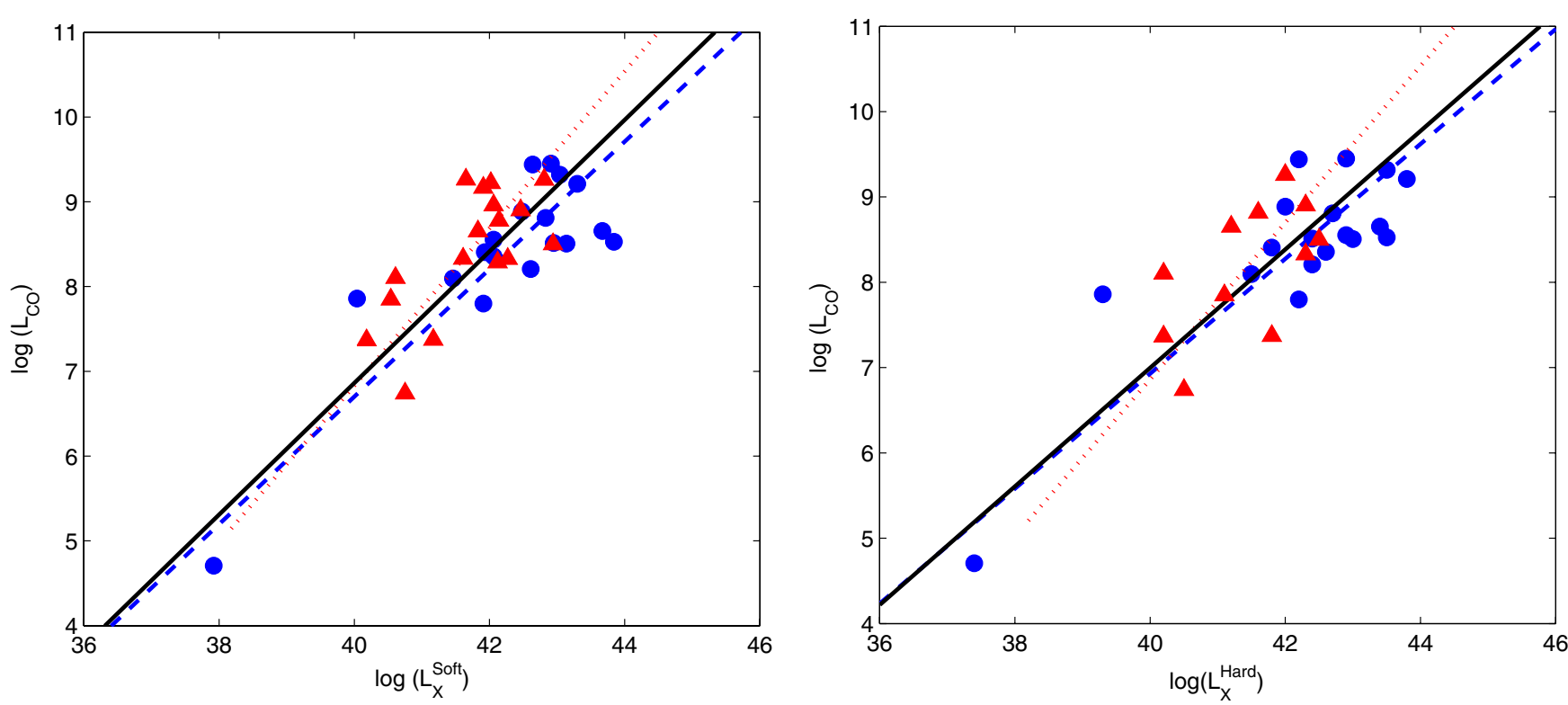

Figure 6. Left: CO luminosity $\left(\mathrm{K} \mathrm{km} \mathrm{s}^{-1} \mathrm{pc}^{2}\right)$ vs. soft $(0.1-2.4 \mathrm{keV}) \mathrm{X}$-ray luminosity ( $\mathrm{erg} \mathrm{s}^{-1}$ ) for the observed Sy1s (circles) and Sy2s (triangles). Right: CO luminosity $\left(\mathrm{K} \mathrm{km} \mathrm{s}^{-1} \mathrm{pc}^{2}\right)$ vs. hard $(0.3-8 \mathrm{keV}) \mathrm{X}$-ray luminosity $\left(\mathrm{erg} \mathrm{s}^{-1}\right)$. Least-squares fits to the data are indicated by the blue dashed (Sy1s), red dotted (Sy2s), and solid (both Sy1s and Sy2s) lines. The correlation factor for each group and null probability are shown in Table 3.

(A color version of this figure is available in the online journal.)

Studying the relation between the CO and FIR luminosities can give us a better understanding of the origin of FIR emission in Seyfert galaxies, and the relationship between the AGN activity, the star formation, and the interstellar medium via the molecular gas content. Taking the average value of $L_{\mathrm{CO}} / L_{\mathrm{FIR}}$ for both Seyfert types we obtain the following result:

$$
\begin{aligned}
\frac{L_{\mathrm{CO}}}{L_{\mathrm{FIR}}}(\mathrm{Sy} 1) & \approx 1.33 \pm 0.13 \times \frac{L_{\mathrm{CO}}}{L_{\mathrm{FIR}}}(\mathrm{Sy} 2) \\
& \approx 3.21 \times 10^{-8} \mathrm{~K} \mathrm{~km} \mathrm{~s}^{-1} \mathrm{kpc}^{2} L_{\odot}^{-1} .
\end{aligned}
$$

There is not a significant difference in the total molecular gas content between the two Seyfert types. This result is consistent with the one obtained by Maiolino et al. (1997) establishing that the total amount of molecular gas is not responsible for the enhanced star formation activity observed in Seyfert 2 hosts (Gonzalez-Delgado \& Perez 1993).

Another interesting observational result from this survey is the strong correlation between the X-ray and the CO luminosity (see Figure 6) with a null probability of $1.83 \times 10^{-5}$ and a correlation coefficient of 0.79 . A least-squares fit yields a fitted relation of $\log \left(L_{\mathrm{CO}}\right)=1 \times \log \left(L_{X}\right)-33.43$ when considering both Seyfert types of our sample. To rule out the possibility of a correlation driven by the luminosity distance $\left(D^{2}\right)$, we study the correlation between $L_{\mathrm{CO}} / L_{\mathrm{FIR}}$ and $L_{X} / L_{\mathrm{FIR}}$, and between $L_{\mathrm{CO}} / L_{X}$ and $L_{X}$; in both cases we get rid of the distance dependence, if any, and find a significant correlation in both cases.

\section{SUMMARY AND DISCUSSION}

We have analyzed the gas properties toward the galaxy nuclei of 18 Seyfert 1 and 17 Seyfert 2 galaxies, taken from the ROSAT/ IRAS sample generated by Condon et al. (1998). The CO line is a molecular gas tracer and provides information about the kinematics of the inner galactic disks through the line width. We investigate the $\mathrm{CO}$ kinematics and find that the $\mathrm{CO}$ line width moderately correlates with the host galaxy inclination for both types of Seyfert galaxies (Figure 2). This may initially suggest that the $\mathrm{CO}$ is coplanar with the galactic disk. However, Heckman et al. (1989) showed that the CO emission may also be correlated with the torus. Studies on the relative orientation of a radio jet and the host galactic disk in Seyfert galaxies, e.g., Schmitt et al. (2002) and Nagar \& Wilson (1999), have shown that the torus is not coplanar with the galactic disk. The combination of these studies suggests that a small, though not negligible, fraction of the $\mathrm{CO}$ emission is coming from the nuclear regions and not solely from the galactic disk. We examine the relation between the CO kinematics and the X-ray power and find a significant correlation between the $\mathrm{CO}$ line widths and the hard X-ray luminosities in both AGN types. Based on the assumption of Eddington accretion, we analyze the correlation between the dynamical mass calculated from the $\mathrm{CO}$ line width and the BHM calculated from the $L_{X}$ for Seyfert galaxies. The measured correlation corroborates the recent studies that suggest that the inclination-corrected $\mathrm{CO}$ line width is a surrogate for the bulge velocity dispersion of the host galaxies (Shields et al. 2006; Wu 2007), and the $\mathrm{BH}$-bulge relation is obtained with this assumption (Ferrarese \& Merritt 2000). As shown in Figure 4, the Eddington limit approximation in our calculations underestimates the $M_{\mathrm{BH}}$ by at least a factor of 10 in comparison to the $M_{\mathrm{BH}}-M_{\text {bulge }}$ relation for normal galaxies where $\left\langle M_{\mathrm{BH}} / M_{\text {bulge }}\right\rangle \sim 0.001$ (Marconi \& Hunt 2003). In order to get a better understanding of the $M_{\mathrm{BH}}-W_{\mathrm{CO}}$ relation for Seyfert galaxies, we have to rely on more accurate measurements of the $M_{\mathrm{BH}}$ and higher resolution images of $\mathrm{CO}$ luminosity in future studies.

Yamada (1994) studied the X-ray $(0.5-4.5 \mathrm{keV})$ and CO luminosity relation in a sample of 13 Seyfert $1 \mathrm{~s}$ and 5 quasars and found a significant correlation. That study suggested a scenario where the star formation activities directly control the mass accretion rate at the central $\mathrm{BH}$, concluding that the more powerful monsters live in the more actively star-forming host galaxies. We analyze the $L_{\mathrm{CO}}-L_{X}$ relation with a more extended sample using both Seyfert types and the X-ray bands $0.1-2.4 \mathrm{keV}$ and $0.3-8 \mathrm{keV}$ and find similar correlations between the X-ray and 
CO luminosities for both Seyfert types. Two possible scenarios can be considered in the nuclear-activity-totalmolecular-gas content relation. First, circumnuclear star formation activity could drive gas into the innermost galactic regions to feed a $\mathrm{BH}$, thus creating a star formation/AGNs connection (Norman \& Scoville 1988; Heckman et al. 1989). The CO emission can be related to the infalling interstellar gas feeding the accretion disk of the massive BHs, which is mostly in molecular form located in the central kiloparsecs. Second, the more powerful $\mathrm{X}$-rays from the central $\mathrm{BH}$ could provide additional heat to the molecular cloud, enhancing the excitation of $\mathrm{CO}$ molecules in the inner region (few parsecs) and enhancing the total CO luminosity. However, the $\mathrm{CO}$ emission observed in our sample with CSO typically comes from inner regions of the galaxy disks $(2-20 \mathrm{kpc})$, as determined from the available interferometer data (e.g., Taylor et al. 1999; Israel 2009; Casasola et al. 2008), while most of the X-ray luminosities likely come from the central engine region (a few pc to $100 \mathrm{pc}$ at most). To establish a clear causal connection between molecular gas in the inner $1 \mathrm{kpc}$ and the fueling of nuclear activity will require higher resolution maps of the $\mathrm{CO}$ emission. A forthcoming Atacama Large Millimeter Array observation will provide the necessary spatial resolution (Maiolino 2008), which will enable us to obtain much accurate kinematics, extended maps of the distribution, and dynamics of the molecular gas.

The authors thank the CSO staff for their support during observations. We thank the anonymous referees for valuable suggestions that improved the manuscript. We are grateful for interesting discussions with Nick Scoville, Martin Emprechtinger, and Tom Bell. The CSO is founded by the National Science Foundation under the contract AST-08388361. This research has made use of the NASA/IPAC Extragalactic Database (NED), which is operated by the Jet Propulsion Laboratory, California Institute of Technology, under contract with the National Aeronautics and Space Administration.

Facilities: CSO, ROSAT, IRAS, CXO

\section{APPENDIX}

\section{CO LINE LUMINOSITY}

We have calculated the $\mathrm{CO}$ line luminosity using the integrated line intensity $(T \Delta V)$, the intensity $(I)$, and the flux $\left(S_{\mathrm{CO}}\right)$. The calculated parameters for each source are listed in Table 2.

To convert from integrated intensity in units of $\mathrm{K} \mathrm{km} \mathrm{s}^{-1}$ into line intensities in units of $\mathrm{erg} \mathrm{cm}^{-2} \mathrm{~s}^{-1} \mathrm{sr}^{-1}$, we use the Rayleigh-Jeans relation,

$$
I\left(\mathrm{erg} \mathrm{cm}{ }^{-2} \mathrm{~s}^{-1} \mathrm{sr}^{-1}\right)=\frac{2 k_{B} \times v^{3}}{c^{3}} \times \frac{T \Delta V\left(\mathrm{~K} \mathrm{~km} \mathrm{~s}^{-1}\right)}{B_{\text {eff }}} \times 10^{6},
$$

where $c$ is the speed of light, $k_{b}$ is the Boltzmann constant, $B_{\text {eff }}$ is main beam efficiency, and $v$ is the line frequency in $\mathrm{GHz}$.
Flux in $\mathrm{Jy} \mathrm{km} \mathrm{s}^{-1}$ is derived as follows:

$$
S\left(\mathrm{Jy} \mathrm{km} \mathrm{s}^{-1}\right)=I \times \frac{c}{v} \times \Omega_{b} \times\left(\frac{v_{0}}{v}\right)^{2} \times 10^{20},
$$

where $\Omega_{b}$ is the solid angle in sr. For a Gaussian beam, the solid angle is given by

$$
\Omega_{b}=1.1333 \times B^{2} \times \frac{1}{206265^{2}} \mathrm{sr},
$$

where $B$ is the half-power beam width in arcseconds.

We calculate the $\mathrm{CO}$ luminosities using the formula in Solomon et al. (1997),

$$
L_{\mathrm{CO}}=\left(c^{2} / 2 k\right) \times S_{\mathrm{CO}} \times \Delta V \times v_{\mathrm{obs}}^{-2} \times D_{L}^{2} \times(1+z)^{-3},
$$

where $D_{L}$ is the luminosity distance ${ }^{7}$ in Mpc.

\section{REFERENCES}

Boller, Th., Meurs, E. J. A., Brinkmann, W., et al. 1992, A\&A, 261, 57 Casasola, V., Combes, F., García-Burillo, S., et al. 2008, A\&A, 490, 61 Condon, J. J., Yin, Q. F., Thuan, T. X., \& Boller, Th. 1998, AJ, 116, 2682 Curran, S. J. 2000, A\&A, 144, 271

Edelson, R. A., Malkan, M. A., \& Rieke, G. H. 1987, ApJ, 321, 233

Ferrarese, L., \& Merritt, D. 2000, ApJ, 529, L9

Gonzalez-Delgado, R. M., \& Perez, E. 1993, Ap\&SS, 205, 127

Graham, A. W., Erwin, P., Caon, N., \& Trujillo, I. 2001, ApJ, 563, L11

Heckman, T. M., Blitz, L., Wilson, A. S., Armus, L., \& Miley, G. K. 1989, ApJ, 342,735

Helou, G., Khan, I. R., Malek, L., \& Boehmer, L. 1988, ApJS, 68, 151

Ho, L. C. 2007, ApJ, 669, 821

Isobe, T., Feigelson, E. D., Akritas, M. G., \& Babu, G. J. 1990, ApJ, 364, 104

Israel, F. P. 2009, A\&A, 493, 525

Kalberla, P. M. W., Burton, W. B., Hartmann, D., et al. 2005, A\&A, 440, 775

Kawakatu, N., Saitoh, T. R., \& Wada, K. 2005, ApJ, 628, 129

Maiolino, R. 2008, New Astron. Rev., 52, 339

Maiolino, R., Ruiz, M., Rieke, G. H., \& Keller, L. D. 1995, ApJ, 446, 561

Maiolino, R., Ruiz, M., Rieke, G. H., \& Papadopoulos, P. 1997, ApJ, 485, 552

Marconi, A., \& Hunt, L. K. 2003, ApJ, 589, L21

Marconi, A., Risaliti, G., Gilli, R., et al. 2004, MNRAS, 351, 169

Nagar, N. M., \& Wilson, A. S. 1999, ApJ, 516, 97

Norman, C., \& Scoville, N. 1988, ApJ, 332, 124

Osterbrock, D. E., \& Ferland, G. J. 2000, Astrophysics Of Gaseous Nebulae and Active Galactic Nuclei (2nd ed.; GBR: Palgrave Macmillan Limited)

Rickard, L. J., \& Harvey, P. M. 1984, AJ, 89, 1520

Rigopoulou, D., Lawrence, A., White, G. J., Rowan-Robinson, M., \& Church, S. E. 1996, A\&A, 305, 747

Sanders, D. B., Schoville, N. Z., \& Soifer, B. T. 1991, ApJ, 370, 158

Schmitt, H. R., Pringle, J. E., Clarke, C. J., \& Kinney, A. L. 2002, ApJ, 575, 150

Shields, G. A., Menezes, K. L., Massart, C. A., \& Vanden Bout, P. 2006, ApJ, 641,683

Solomon, P. M., Downes, D., Radford, S. J. E., \& Barrett, J. W. 1997, ApJ, 478, 144

Taylor, C. L., Hüttemeister, S., Kein, U., \& Greve, A. 1999, A\&A, 349, 424

Tully, R. B., \& Fisher, J. R. 1977, A\&A, 54, 661

Wu, X. B. 2007, ApJ, 657, 177

Yamada, T. 1994, ApJ, 423, L27

Young, J. S., Schloerb, F., Kenney, J. D., \& Lord, S. D. 1986, ApJ, 304, 443

\footnotetext{
7 For all the sources, we obtained the luminosity distance using the Web site calculator of http://www.astro.ucla.edu/ wright/CosmoCalc.html, using a cosmology of $H_{0}=77 \mathrm{~km} \mathrm{~s}^{-1} \mathrm{Mpc}^{-1}, \Omega_{M}=0.27$, and $\Omega_{V}=0.73$.
} 